\title{
Radar observations of field-aligned plasma irregularities in the SEEK-2 campaign
}

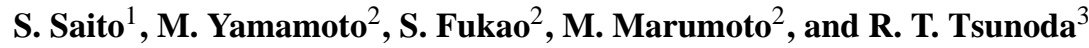 \\ ${ }^{1}$ National Institute of Information and Communication Technology, Nukui-Kita 4-2-1, Koganei, Tokyo 184-8795, Japan \\ ${ }^{2}$ Research Institute of Sustainable Humanosphere, Kyoto University, Gokasho, Uji, Kyoto 611-0011, Japan \\ ${ }^{3}$ SRI International, Menlo Park, California, USA
}

Received: 18 January 2005 - Revised: 12 May 2005 - Accepted: 18 May 2005 - Published: 13 October 2005

Part of Special Issue "SEEK-2 (Sporadic-E Experiment over Kyushu 2)"

\begin{abstract}
During the Sporadic E Experiment over Kyushu 2 (SEEK-2) campaign, field-aligned irregularities (FAIs) associated with midlatitude sporadic-E $\left(E_{s}\right)$ layers were observed with two backscatter radars, the Lower Thermosphere Profiler Radar (LTPR) and the Frequency Agile Radar (FAR), which were located $40 \mathrm{~km}$ apart in Tanegashima, Japan. We conducted observations of FAI echoes from 31 July to $24 \mathrm{Au}-$ gust 2002, and the radar data were used to determine launch timing of two sounding rockets on 3 August 2002. Our comparison of echoes obtained by the LTPR and the FAR revealed that echoes often appeared at the FAR about $10 \mathrm{~min}$ earlier than they did at the LTPR and were well correlated. This indicates that echoing regions drift with a southward velocity component that maintains the spatial shape. Interferometry observations that were conducted with the LTPR from 3 to 8 August 2002, revealed that the quasi-periodic (QP) striations in the Range-Time-Intensity (RTI) plots were due to the apparent motion of echoing regions across the radar beam including both main and side lobes. In most cases, the echo moved to the east-southeast at an almost constant altitude of $100-110 \mathrm{~km}$, which was along the locus of perpendicularity of the radar line-of-sight to the geomagnetic field line. We found that the QP pattern on the RTI plot reflects the horizontal structure and motion of the $E_{s}$ layer, and that echoing regions seemed to be in one-dimensionally elongated shapes or in chains of patches. Neutral wind velocities from 75 to $105 \mathrm{~km}$ altitude were simultaneously derived with meteor echoes from the LTPR. This is the first time-continuous simultaneous observation FAIs and neutral wind with interferometry measurements. Assuming that the echoing regions were drifting with an ambient neutral wind, we found that the echoing region was aligned east-northeastwest-southwest in eight out of ten QP echo events during the SEEK-2 campaign. A range rate was negative (positive),
\end{abstract}

Correspondence to: S. Saito

(susaito@nict.go.jp) when a frontal structure of echoing regions elongated eastnortheast-west-southwest drifts with southward (northward) neutral wind.

Keywords. Ionosphere-atmosphere interactions; Ionospheric irregularities; Plasma waves and instabilities

\section{Introduction}

There is a general introduction to this Sporadic E Experiment over Kyushu 2 (SEEK-2) campaign by Yamamoto et al. (2005). The primary purpose of the radar experiment was to monitor field-aligned irregularities (FAIs) in the ionospheric $E$-region around rocket flight paths and determine launch timing. The results obtained by radar observations were immediately transferred to the control room of the launch site via a network. We added interferometer capabilities to one of our radars to image the fine structures of FAIs and to derive neutral wind velocities by using meteor echoes. This is the first attempt to observe FAIs and neutral wind velocity simultaneously with interferometry measurements.

Quasi-periodic (QP) echoes are characterized by striations in the Range-Time-Intensity (RTI) plots and the quasiperiodic variation of echo intensity in time with $5-10 \mathrm{~min}$. Since QP echoes were found by Yamamoto et al. (1991) using the Middle and Upper Atmosphere (MU) radar, several theories have been proposed to explain the mechanism behind them. Woodman et al. (1991) pointed out that atmospheric gravity waves could modulate $E_{s}$ layers to keep the plasma unstable and could explain the quasi-periodicity. Tsunoda et al. (1994) proposed a model that $E_{s}$ layers deeply modulated in altitude by atmospheric gravity waves could explain both the striation in the RTI plots and the quasiperiodicity. Modulation of $E_{s}$ layers in altitude extent of a few kilometers were observed by Miller and Smith (1978) by using the Arecibo incoherent scatter radar. However, 
Table 1. Specifications for the FAR and the LTPR

\begin{tabular}{lll}
\hline & FAR & LTPR \\
\hline Center Frequency & $24.515 \mathrm{MHz}$ & $31.57 \mathrm{MHz}$ \\
Peak Power & $4 \mathrm{~kW}$ & $20 \mathrm{~kW}$ \\
Antennae & 4 -element Yagi $\times 8$ & 4 -element Yagi $\times 9$ \\
Pulse code & 13 -bit Barker & 16 -bit complementary \\
Number of Receiver Channels & 1 & 6 \\
Beam width $(-3 \mathrm{~dB}$, one way) & $6^{\circ}$ & $10^{\circ}$ \\
\hline
\end{tabular}

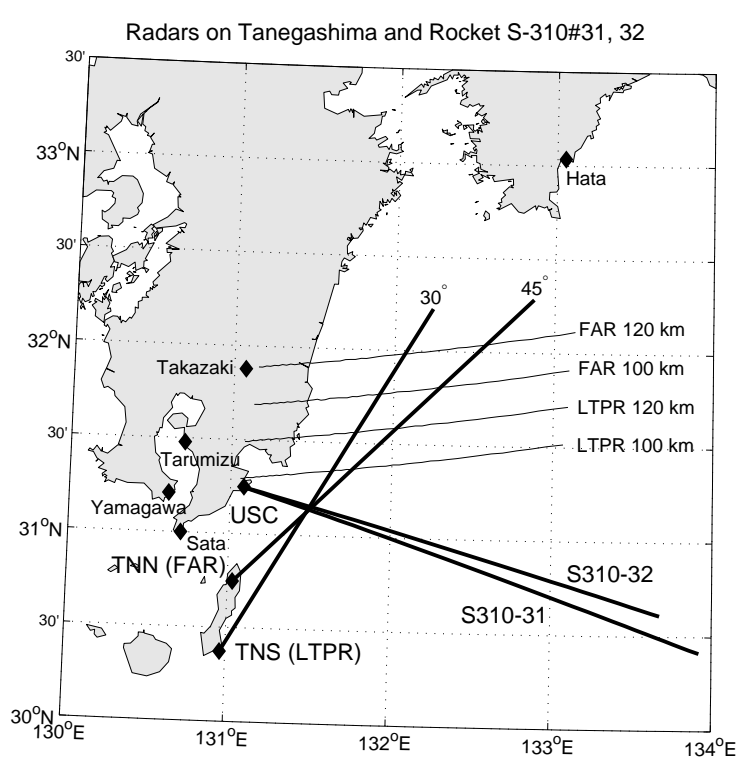

Fig. 1. Arrangement of radar sites, launch site, area observed by radars and rockets. TNS, TNN, and USC stand for Tanegashima South site, Tanegashima North site, and Uchinoura Space Center, respectively.

deeply-modulated $E_{S}$ layers were not found through in-situ observations during the previous SEEK campaign (Fukao et al., 1998). Using an ionosonde, Ogawa et al. (2002) found that $E_{s}$ layers, where the MU radar detects QP echoes, were not largely distorted in altitude.

Maruyama et al. (2000) proposed that inhomogeneous $E_{S}$ layers and external electric field can generate polarization electric fields, which can invoke image striations of irregularities along the geomagnetic field line, and these may invoke gradient-drift plasma instability. Relative motion of unstable regions along the geomagnetic field line to an observer could result in characteristic striations in RTI plots. Results obtained through computer simulations that support this theory including the effects of neutral wind were recently published by Yokoyama et al. (2003a,b). Larsen (2000) associated the QP echoes with patchy structure in $E_{S}$ layers and pointed out that neutral wind shear instabilities and the associated Kelvin-Helmholtz structure could play an important role in seeding the gradient-drift instability resulting in radar backscatter. Hysell and Burcham (2000) proposed a model where irregularities in randomly distributed unstable plasma patches or blobs in $E_{s}$ layers could produce QP structures due to the effect of refracted radar waves and finite aspect angle sensitivity of irregularities. Wang and Chu (2001) also made observations that supported this model using Chung-Li VHF radar. Although there have been many discussions on the generation mechanism of QP echoes, it has not yet been satisfactorily explained.

Using the MU radar and an ionosonde, Ogawa et al. (2002) found that FAI echoes could be observed when $\mathrm{f}_{o} E_{s}-\mathrm{f}_{b} E_{s}$ exceeded $1 \mathrm{MHz}$, where $\mathrm{f}_{o} E_{s}$ and $\mathrm{f}_{b} E_{s}$ were the maximum reflected and the minimum transparent frequencies in the $E_{S}$ layer, respectively. Since $\mathrm{f}_{o} E_{s}$ and $\mathrm{f}_{b} E_{s}$ correspond to the highest and lowest plasma density in the $E_{S}$ layer, respectively, they pointed out that the localized plasma density gradient within the $E_{S}$ layer play an important role, which is consistent both with suggestions of both Maruyama et al. (2000) and Hysell and Burcham (2000). Hysell et al. (2002) found that it was necessary to take into account what effect the refraction of radar waves by dense plasma clouds had in order to explain how QP striations were produced. They also pointed out that echoes resulted from wide azimuth angles to explain very long striations up to $60 \mathrm{~km}$ in range extent.

Cosgrove and Tsunoda (2002) proposed $E_{s}$-layer instability, which is at its strongest when the phase front of $E_{S}$ layer distortion is northwest-southeast (see also Tsunoda et al., 2004). Bernhardt (2002) showed that Kelvin-Helmholtz instability in the neutral atmosphere could modulate $E_{S}$ layers into a quasi-periodic structure. Neutral winds have been assumed to be not only a source of polarization electric field but also a source of the quasi-periodic nature of QP echoes. We used two ionospheric backscatter radars for this study; the first was capable of an interferometry experiment enabling us to investigate the spatial structure of QP echoes. By observing meteor echoes with QP echoes, neutral wind velocity around the $E_{s}$ layers were derived simultaneously.

\section{Observation setup}

We installed two ionospheric radars on Tanegashima Island, which is located to the south of the Uchinoura Space Center (USC) as shown in Fig. 1. The first was a Lower Thermosphere Profiler Radar (LTPR) operating at $31.57 \mathrm{MHz}$, and 
Table 2. Occurrence of FAI echoes for the LTPR. QP, CN, LQP, and UD stand for QP-type, Continuous-type, Low-altitude QP, and Undefined (structured but not quasi-periodic), respectively. " $\sqrt{ }$ " means that the particular type of echoes were observed on the particular day. No data were available on 5 August and 15 due to system trouble.

\begin{tabular}{|c|c|c|c|c|c|c|c|c|c|c|c|c|c|}
\hline Date & 31 July & 1 August & 2 & 3 & 4 & 5 & 6 & 7 & 8 & 9 & 10 & 11 & 12 \\
\hline QP & & & & $\sqrt{ }$ & $\sqrt{ }$ & - & $\sqrt{ }$ & $\sqrt{ }$ & & $\sqrt{ }$ & $\sqrt{ }$ & $\sqrt{ }$ & $\sqrt{ }$ \\
\hline $\mathrm{CN}$ & & & & & & - & & & $\sqrt{ }$ & & & & \\
\hline LQP & & & & & & - & & & & & & & \\
\hline UD & $\sqrt{ }$ & $\sqrt{ }$ & $\sqrt{ }$ & & $\sqrt{ }$ & - & $\sqrt{ }$ & & $\sqrt{ }$ & $\sqrt{ }$ & $\sqrt{ }$ & & \\
\hline Date & 13 & 14 & 15 & 16 & 17 & 18 & 19 & 20 & 21 & 22 & 23 & 24 & total \\
\hline $\mathrm{QP}$ & $\sqrt{ }$ & & - & $\sqrt{ }$ & $\sqrt{ }$ & $\sqrt{ }$ & $\sqrt{ }$ & $\sqrt{ }$ & $\sqrt{ }$ & $\sqrt{ }$ & & $\sqrt{ }$ & $17 / 23$ \\
\hline $\mathrm{CN}$ & & & - & & $\sqrt{ }$ & & $\sqrt{ }$ & & & & & & $3 / 23$ \\
\hline LQP & & & - & & & & & & & & & & $0 / 23$ \\
\hline UD & & $\sqrt{ }$ & - & & $\sqrt{ }$ & & & $\sqrt{ }$ & & & $\sqrt{ }$ & & $12 / 23$ \\
\hline
\end{tabular}

the second was a Frequency Agile Radar (FAR) operating at 24.515 MHz. The specifications for the two radars are listed in Table 1.

The LTPR was installed in Tanegashima Space Center (TNSC), the Japan Aerospace Exploration Agency (JAXA) $\left(130.96^{\circ} \mathrm{E}, 30.38^{\circ} \mathrm{N}\right)$, which we call "Tanegashima South site (TNS)". The LTPR has six receiver channels that enable interferometer observations. We arranged its antennae so that both radar imaging and meteor echo measurement were possible. The antenna array consisted of nine Yagi antennae in a linear and a triangular arrays as shown in Fig. 2. The transmitting antennae are in the linear array pointed to $30^{\circ}$ to the east from geographic north. The declination angle of the geomagnetic field around the observation region is about $-5^{\circ}$. Six antennae, $1,2,5,7,8$, and 9 , formed the receiving array. The triangular array (antennae 1, 2, and 9) was used as a simple interferometer to determine the arrival angles for both the FAI echoes and meteor echoes. Aliasing in space is inevitable, as the shortest distance between antennae is $9.5 \mathrm{~m}$ ( $\sim 1$ wavelength). The antenna patterns for the LTPR are plotted in Fig. 3. Figure 3 (a) is the two-way antenna pattern where the six receiving antennae are considered. Figure 3 (b) is the two-way pattern where a single antenna is used for receiving. This pattern should apply for interferometry measurements where each antenna is used independently. The LTPR provided information on the occurrence of FAIs which was the most important condition for the rocket launches. Interferometry observations are supposed to provide detailed spatial structures of FAIs.

The FAR was installed in the Tanegashima Meadow of Nishinoomote City $\left(131.03^{\circ} \mathrm{E}, 30.75^{\circ} \mathrm{N}\right)$, which we call "Tanegashima North site (TNN)". The system had only one receiving channel and its main transmitting beam was pointed to $45^{\circ}$ to the east from the geographic north. The objective of this radar was to determine large scale distribution of FAIs through the LTPR observation as well as to monitor

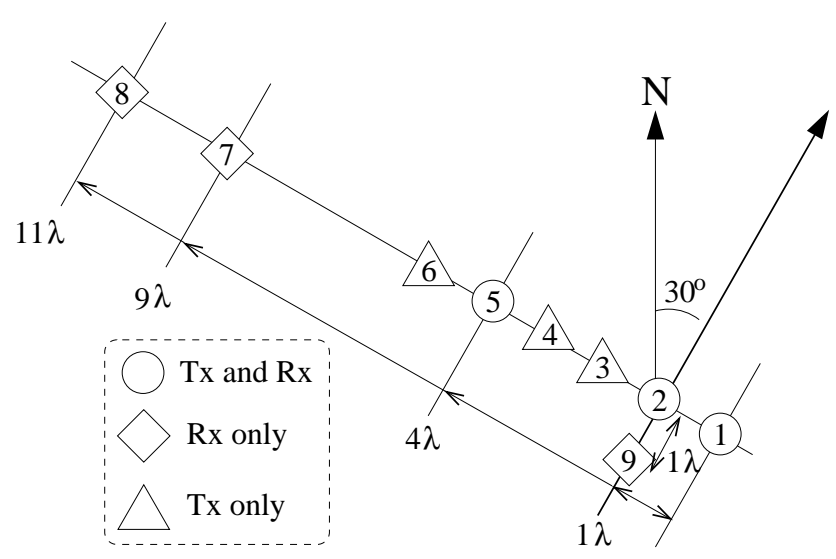

Fig. 2. Antenna layout for the LTPR. Tx array consists of antennae 1-6, while $R x$ array consists of antennae $1,2,5,7,8$, and 9.

FAI occurrences as a back-up to the LTPR.

The observation areas of the LTPR and the FAR are depicted in Fig. 1. Due to high aspect angle sensitivity to the geomagnetic field line, radar echoes were mainly expected between two loci of perpendicularity at altitudes of 100 and $120 \mathrm{~km}$ for each radar. Because of the coherent nature of FAI echoes, echoes may have been detected not only in the radar main lobe but also in the side lobes. We can see that the area observed by these two radars was relatively narrow to the north-south direction and wide to the east-west. It should be noted that the radars did not observe the ionosphere along rocket trajectories, but observed just north of them.

Both radars were operated from 31 July to 24 August 2002. Due to limitations with data storage and trouble with the system, satisfactory interferometry data from the LTPR was only available from 3 to 8 August. 
(a)

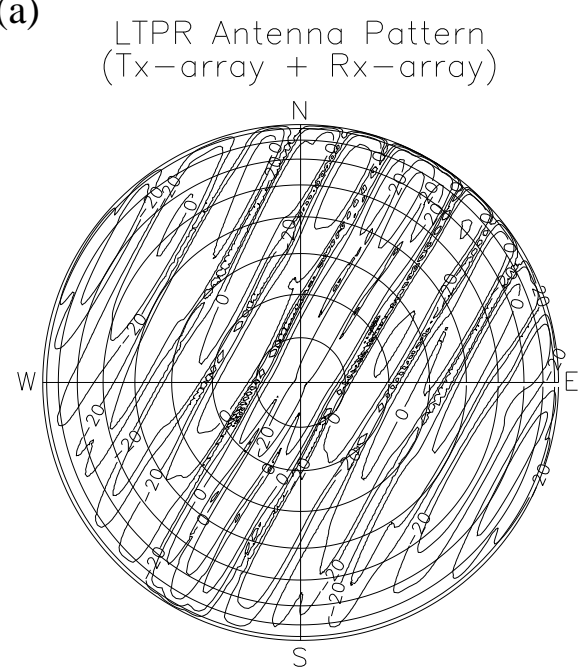

(b)

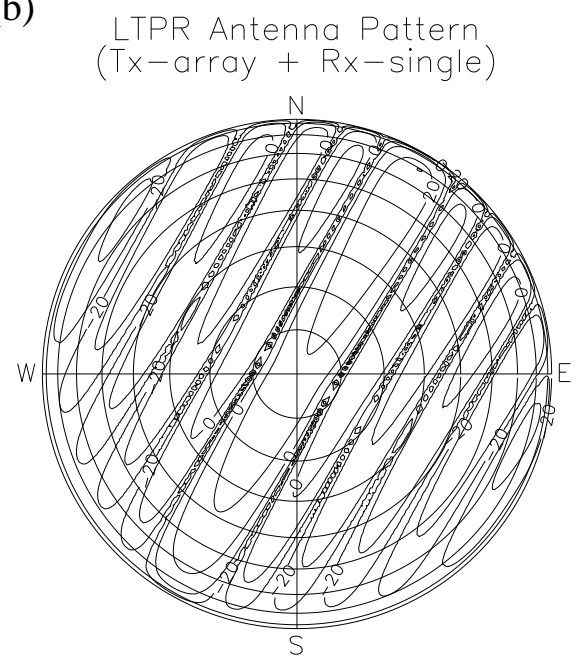

Fig. 3. Two-way antenna patterns for the LTPR, (a) where the $R x$ array is used for receiving, (b) where a single antenna is used for receiving. Concentric circles indicate zenith angles with a $10 \mathrm{deg}$ interval.

\section{FAI echoes observed by LTPR and FAR}

Occurrence of FAI echoes which are categorized in several types observed by the LTPR are summarized in Table 2. QP echoes were observed in 17 nights of 23. Continuous-type echoes were also observed in 3 nights. Low-altitude QP (LQP) echoes were not observed throughout the campaign. There were a lot of echoes with some structures which were not quasi-periodic and categorized in U.D. (Undefined) type.

The echo power measured with the two radars around the rocket launches are in Range-Time-Intensity (RTI) format in Fig. 4. For the LTPR, echo power received by the $R x$ array were plotted. The "apparent altitudes" are shown by the right vertical axes of the RTI plots in Fig. 4 as well as the ranges by the left vertical axes. They are calculated from the ranges which are directly measured and the condition of perpendicularity of the radar k-vector and geomagnetic field (a)

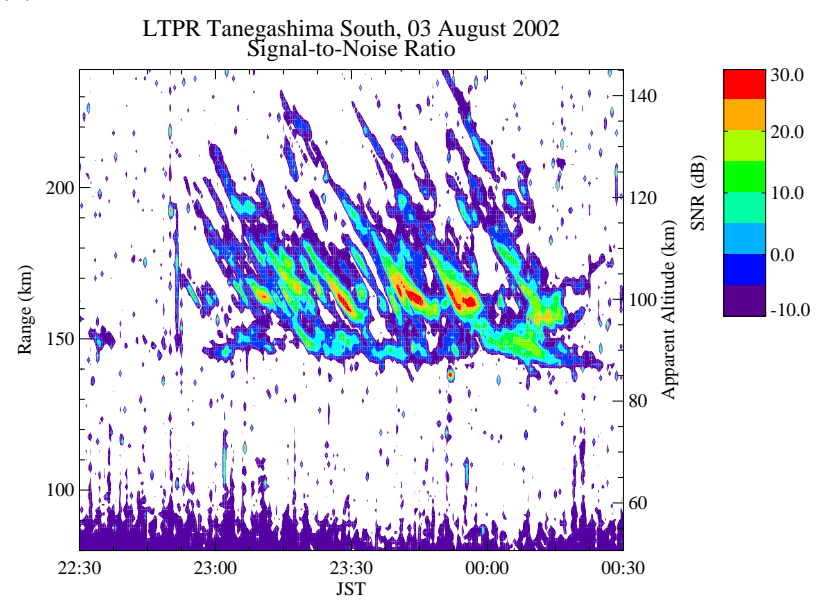

(b)

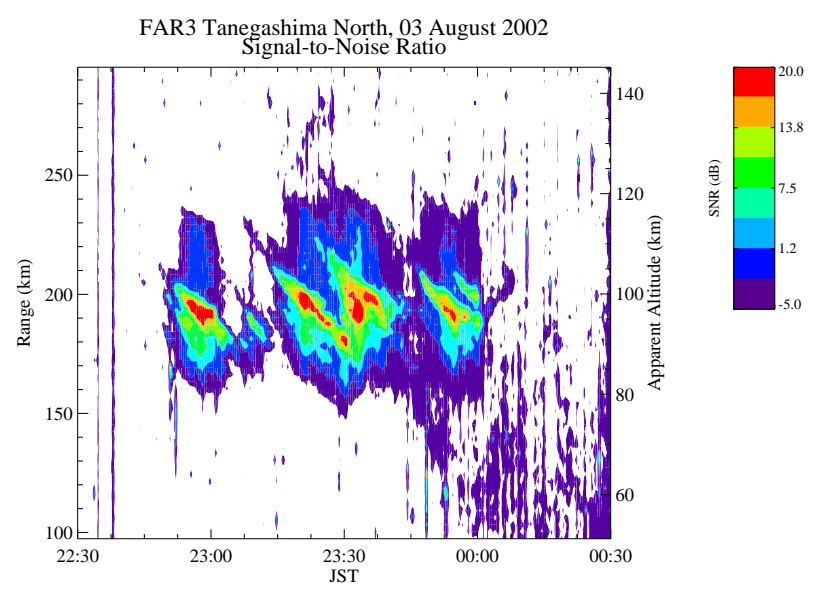

Fig. 4. Range-Time-Intensity plots of echo power observed by (a) LTPR and (b) FAR from 2230 JST 3 August to 0030 JST 4 August 2002. The left vertical axes show the ranges of echoes, while the right axes show the "apparent altitudes" which are calculated from the range and the perpendicularity condition assuming that the azimuth of echoes are at the center of the radar beam $\left(+30^{\circ}\right)$.

in the radar main beam direction. Therefore, the "apparent altitudes" may be different from the "true altitudes". The "true altitude" can be determined by interferometry. The first rocket (S310-31) was launched at 2324 JST $(=\mathrm{UT}+9 \mathrm{~h})$ and the second (S310-32) at 2339 JST. Both the radars observed typical QP echoes for about an hour. The FAR observed echoes from 2245 to 0010 JST, and the LTPR from 2300 to 0030 JST. Both sequences of QP striations looked similar, while there seemed to be some time delay from the FAR to the LTPR. To determine how they were similar and how long the delay was, we calculated the cross-correlations of temporal variations for echo power observed with the FAR and the LTPR at the range of each radar where the average echo power was strongest. We applied a low-pass filter with a cutoff period of $5 \mathrm{~min}$ to the temporal variations for echo power. A maximum correlation of 0.73 was obtained at a $-19.5 \mathrm{~min}$ lag (negative lag means that the FAR observed echoes prior 


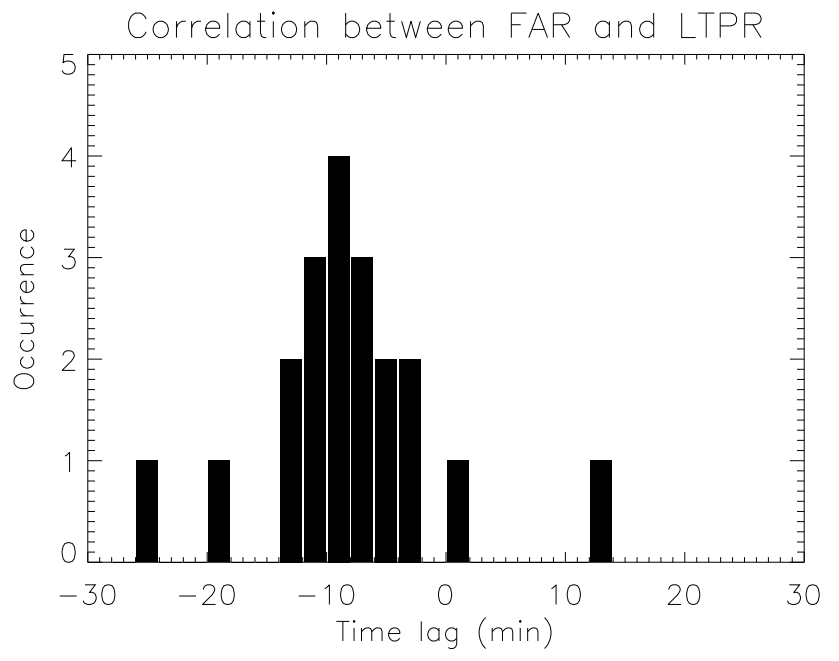

Fig. 5. Distribution of lag time between echoes observed by the LTPR and the FAR. Positive lag means that the LTPR detects echoes prior to the FAR.

to the LTPR). This indicates that there are large-scale structures propagating from the FAR's observation region to the LTPR's, which are horizontally separated by about $80 \mathrm{~km}$. It should be noted that this velocity is apparent phase velocity, since the direction of propagation was not known. We did the same analysis for 20 events where the LTPR and the FAR observed FAI echoes simultaneously during the whole observation period. Figure 5 plots the distribution of lag between the LTPR and the FAR. In most of events, the FAR observed echoes prior to the LTPR by about $10 \mathrm{~min}$. This means that FAI echoing regions propagate to a certain preferential direction. However, the precise propagation directions and velocities cannot be determined with this analysis.

\section{Interferometry observation of FAIs}

We used the triangle array (antennae 1, 2, and 9 in Fig. 2) for the LTPR for interferometry data analysis in this paper. Since the shortest baseline was longer than half the wavelength (about one wavelength), multiple solutions for the arrival angle of an echo could be obtained. However, we could find a unique solution by limiting the range of the solution so that it was close to the directions in which the radar lines-ofsight were perpendicular to the geomagnetic field lines at $E$ region altitudes. We set conditions of $-45^{\circ}<$ Azimuth $<90^{\circ}$ and $0^{\circ}<$ Elevation $<55^{\circ}$ for our analysis. Echoes where the arrival angle could not be uniquely determined even under these conditions were discarded. Because the baseline length was not too long, the arrival angles for most FAI echoes were obtained without ambiguity with this method.

Figure 6 (a) plots the distribution of FAI echoes across the sky. FAI echoes were distributed around the locus of perpendicularity, but widely distributed in the azimuth not only in the radar's main lobe but also in the side lobes. This figure also tells us that echoes at longer ranges, which are of- (a)

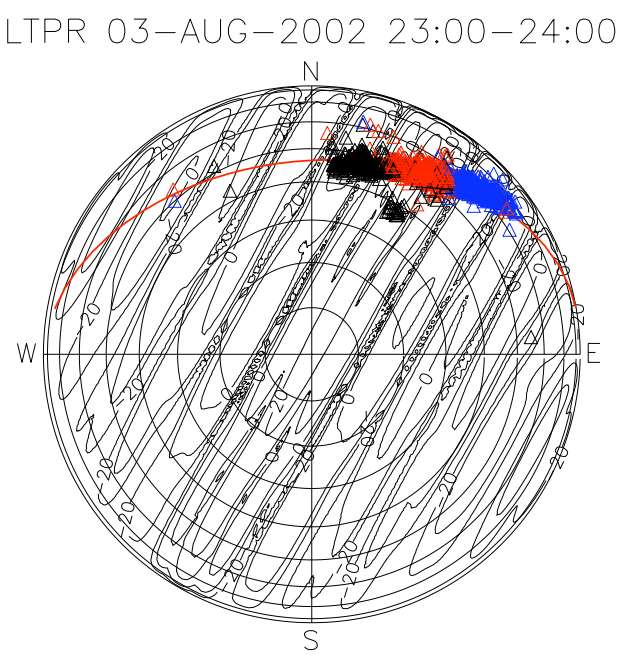

(b)

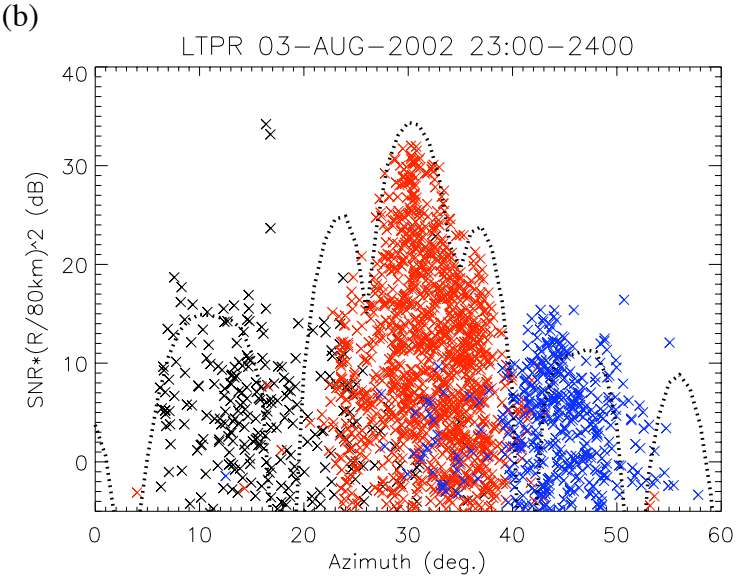

Fig. 6. (a) Distributions of arrival angles for FAI echoes from 2300 to 2400 JST 3 August 2002. Thick red line indicates directions in which line of sight is perpendicular to geomagnetic field line at $100 \mathrm{~km}$ altitude. Colors indicate the ranges of echoes. (Black: Range $\leq 155 \mathrm{~km}$, Red: $155<$ Range $\leq 185 \mathrm{~km}$, Blue: Range $>185 \mathrm{~km}$ ). Beam pattern of the LTPR (two-way) has also been plotted. (b) Distribution of echo power measured by the receiving array over azimuth angle for the echoes plotted in (a). Range correction with respect to the reference range of $80 \mathrm{~km}$ has been done. Colors indicate the ranges of echoes in the same way as in (a). Dashed line shows the shape of the "two-way" antenna pattern in logarithmic scale along the locus of perpendicularity at $100 \mathrm{~km}$ altitude.

ten interpreted as echoes from a higher altitude arrive from the eastern side lobes where elevation angles are low to satisfy geomagnetic perpendicularity conditions. In the same way, echoes at shorter ranges arrive from the western side lobes where elevation angles are high. It can be observed from Fig. 4 (a) that the echo power were decreased at ranges $\sim 155 \mathrm{~km}$ and $\sim 185 \mathrm{~km}$. These "gaps" on the RTI plot correspond to the nulls between the main and side lobes of the LTPR's beam pattern. Figure 6 (b) confirmed that the envelope of the distribution of echo power received by the $R x$ array reproduced the LTPR's beam pattern. 


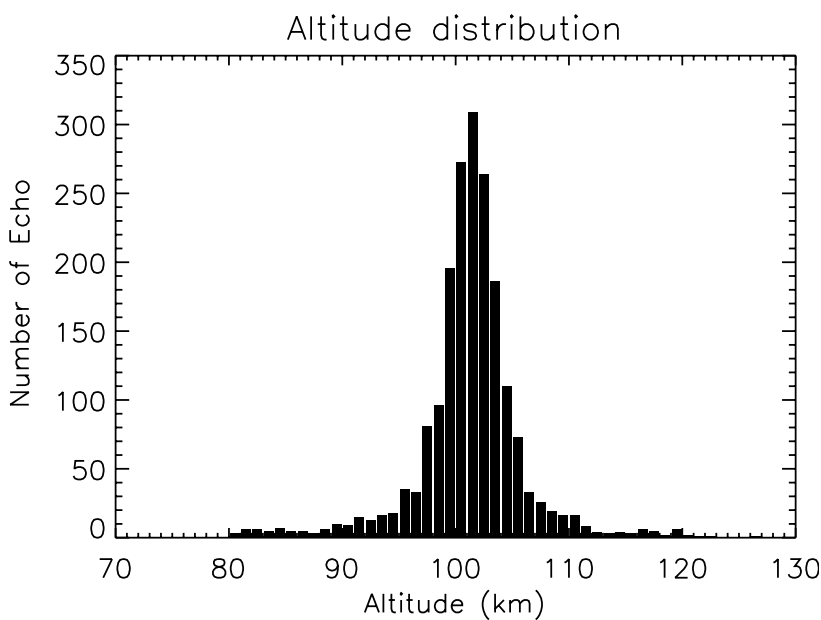

Fig. 7. Histogram of true height distribution of echoes by the LTPR at 2300-2400 JST, 3 August 2002.

Figure 7 plots the distribution for the true height of echoes during the rocket experiment on 3 August determined with the interferometer technique. It is easy to see that most echoes are at altitudes between 95 and $110 \mathrm{~km}$ and there are few echoes with the true heights higher than $120 \mathrm{~km}$. As discussed by Hysell et al. (2002), radar waves may be refracted by steep plasma density gradient around the $E_{S}$ layer up to $\pm 1.5^{\circ}$. However, this does not largely change estimated true heights. Since refraction occur very close to the scattering point which are shown to be around the $E_{s}$ layer, the error of the position owing to the refraction is small. The echo range was not a correct indicator of the echo height, but was a indicator of azimuthal arrival angles. However, weak echoes at higher altitudes (up to $120 \mathrm{~km}$ ) have been detected by MU radar interferometry (Yamamoto et al., 1994). This may be because the MU radar is much more sensitive to the FAI echoes, has a narrower radar beam, and observes in the direction much close to the geomagnetic north compared with the LTPR in the SEEK-2 campaign.

According to what has been discussed above, the echo locations of QP striations with negative range rates should move westward across the LTPR beam pattern, and those with positive range rates should move eastward. To confirm whether this occurred, we tracked the positions of various QP striations observed during the SEEK-2 campaign. Figure 8 (a) is an RTI plot for echo power observed from 23 to 24 JST on 3 August 2002, when the rockets were launched. We tracked two striations in the RTI plot. During this time period, all the striations had negative range rates. These echoes both in Traces 1 and 2 moved westward across the radar beam at an almost constant altitude of $100 \mathrm{~km}$ along the locus of perpendicularity (Fig. 8(b)-(e)). There is another example obtained between 02 and 03 JST on 7 August 2002, in Fig. 9. The range rate changed from negative to positive at 0240 JST. The tracking echo locations of Trace 3 with a negative range rate (Fig. 9(b) and (c)) exhibited westward motion, and Trace 4 with a positive range rate (Fig. 9(d) and (e)) exhibited eastward motion. During this time interval, the true heights of echoes were almost constant at about $100 \mathrm{~km}$.

Because of the high magnetic aspect angle sensitivity of FAIs, their effective radar field-of-view was quite narrow across the locus of perpendicularity and wide along it. Therefore, we concluded that the radar observes echoing regions passing across the effective field-of-view along the locus of perpendicularity like a slit camera does. The QP striations can be explained by assuming one-dimensionally elongated echoing regions passing across the slit. A single striation is often split into several clusters of echoes. For example, Trace 2 in Fig. 8(a) consists of five clusters of echoes. We found that this was not only because the echoes were at the null points of the radar beam pattern. This indicates that patchy echoing regions were organized in a line and drifted horizontally as a group.

In what follows, we discuss how we interpreted the RTI plots of QP echoes. QP striations are apparently produced by the horizontal motion of echoes at an almost constant height. A negative range rate is, for the LTPR beam pattern, interpreted as the motion of the echoing region with a westward velocity component. A positive range rate means an eastward velocity component for the moving echoing region. Gaps consistently seen in all QP striations of the LTPR around the 155 and $185 \mathrm{~km}$ ranges correspond to null points of the antenna pattern. Such gaps in QP striations could not be detected with the FAR. The FAR had a sharper beam, which meant more suppressed side lobes. Therefore, the FAR only detected echoes in the main lobe. This also explains why echoes detected by the FAR were limited in narrower ranges than echoes by the LTPR. If the main lobe had been pointing to geomagnetic north, echoes at shorter ranges would have arrived from the main lobe, and echoes at longer ranges would have arrived from the side lobes. If the main and side lobes had been pointing to the west of geomagnetic north, ascending striations would have been seen in an RTI plot, when descending striations were seen by the radar pointing to the east of the geomagnetic north.

\section{Neutral wind derived from meteor echoes and FAI structure}

The LTPR could detect meteor echoes as well as FAI echoes. Meteor trails are generated at altitudes of lower E and upper D region (70-110 km) and effectively reflect VHF radio waves. As they move with ambient neutral atmosphere, mean horizontal wind velocities can be estimated by finding best fit to observed Doppler velocities of meteor trails. Meteor echoes were distinguished from FAI echoes by their characteristic temporal variations in power. The same antennae that had been set up as for FAI interferometry was used to determine the locations of meteor trails. To determine the unique arrival angles of meteor echoes, we assumed that meteor echoes could be found between 75 and $105 \mathrm{~km}$ altitude. Furthermore, interferometry solutions with azimuth $-45^{\circ}$ $105^{\circ}$ or $180^{\circ}-240^{\circ}$ and elevation angles of less than $60^{\circ}$ were 
(a)

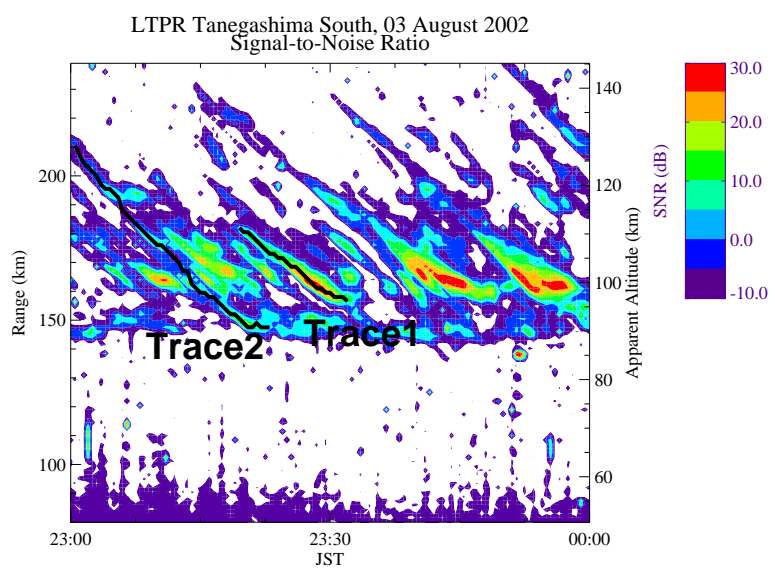

(b)

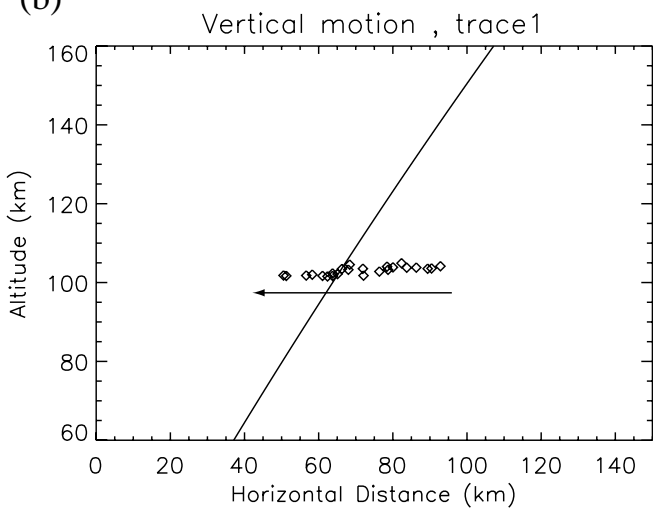

(d)

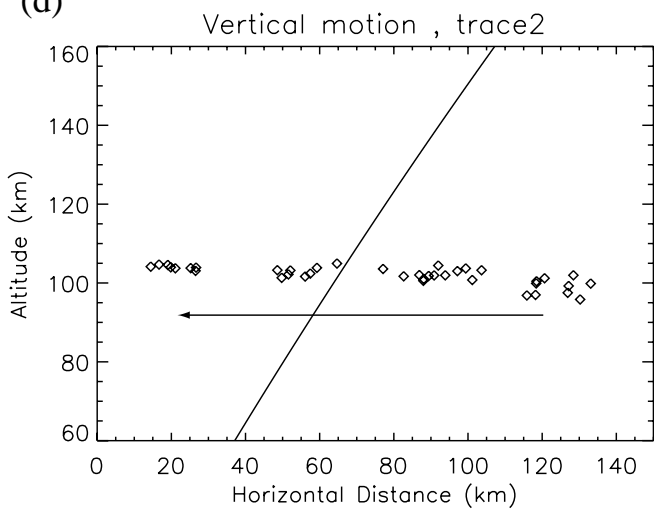

(c)
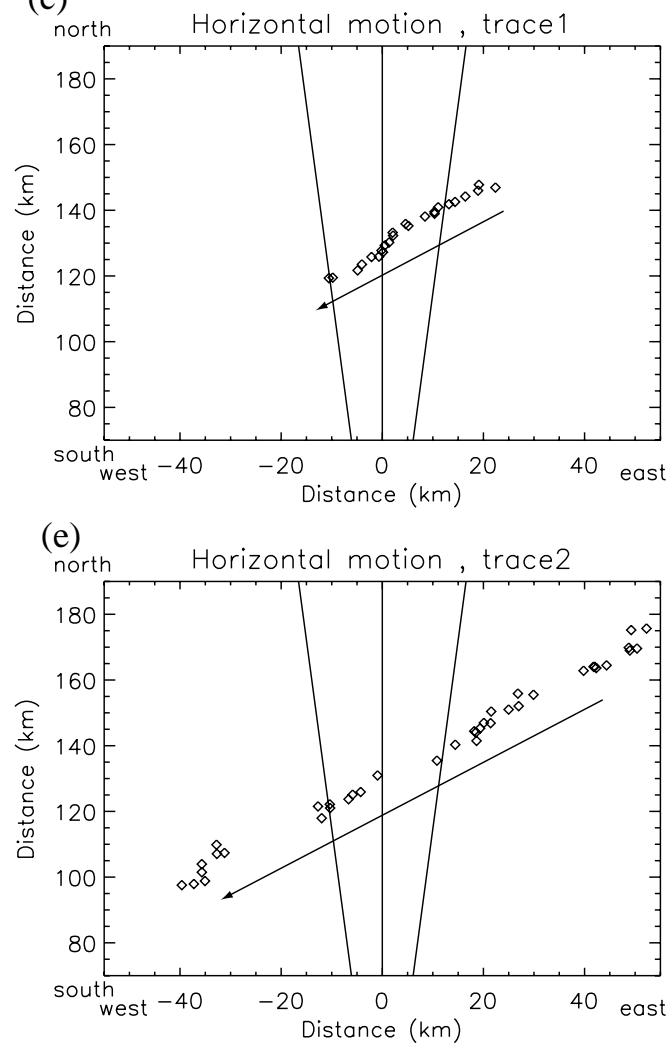

Fig. 8. (a) Echoes observed between 2300 and 2400 JST on 3 August 2002. Motion of echoes on Trace 1 in (a) is tracked in (b) the vertical and (c) horizontal planes. Solid line in (b) indicates the center of radar beam (Azimuth $+30^{\circ}$ ). Three solid lines in (c) indicates the center of radar beam and the directions of $+30 \pm 5^{\circ}$. (d) and (e) are same as (b) and (c), except for Trace 2 in (a).

adopted. For solutions with elevation angles of more than $60^{\circ}$, any azimuth angle was allowed. Echoes whose unique arrival angles could not be determined based on these restrictions were discarded. With meteor echoes selected according to these conditions, we estimated the neutral wind velocity with an altitudinal resolution of 3-6 km and temporal resolution of 1 hour. It should be noted that it is the neutral wind velocity over the LTPR that is derived from the meteor echo measurements, while FAIs are observed along the locus of perpendicularity.
Figure 10 shows the zonal and meridional neutral wind velocity profiles derived from meteor echoes between 2324 LT on 3 August 2002, when the rockets were launced. The neutral wind velocity derived from meteor echoes at 100 $105 \mathrm{~km}$ was $52 \mathrm{~m} / \mathrm{s}^{-1}$ toward $-70^{\circ}$ azimuth. The second rocket (S310-32) released TMA (Tri-Methyl Aluminum) so that neutral wind velocity around the rocket orbit could be measured. The neutral wind velocity at an altitude of $103 \mathrm{~km}$ derived from the TMA trail was $85 \mathrm{~m} / \mathrm{s}^{-1}$ toward $-145^{\circ}$ for the upleg, and $70 \mathrm{~m} / \mathrm{s}^{-1}$ toward $-98^{\circ}$ for the downleg 
(a)

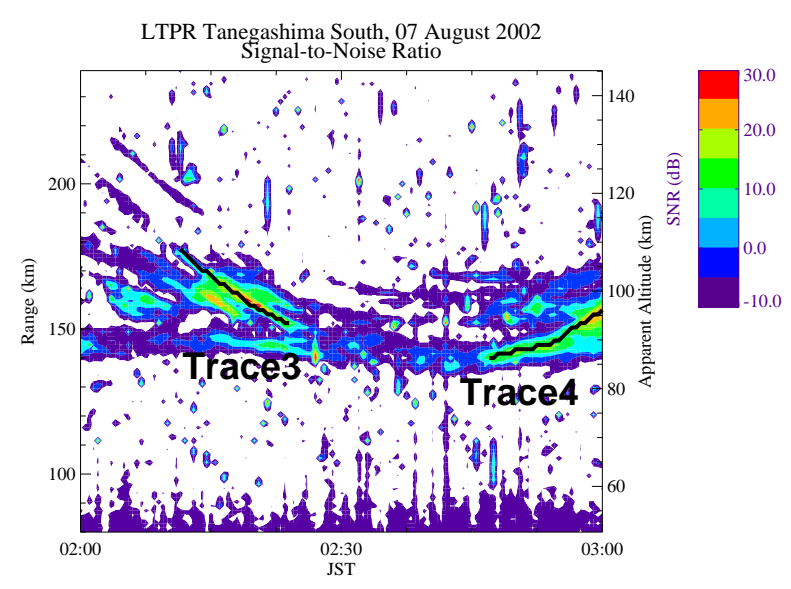

(b)

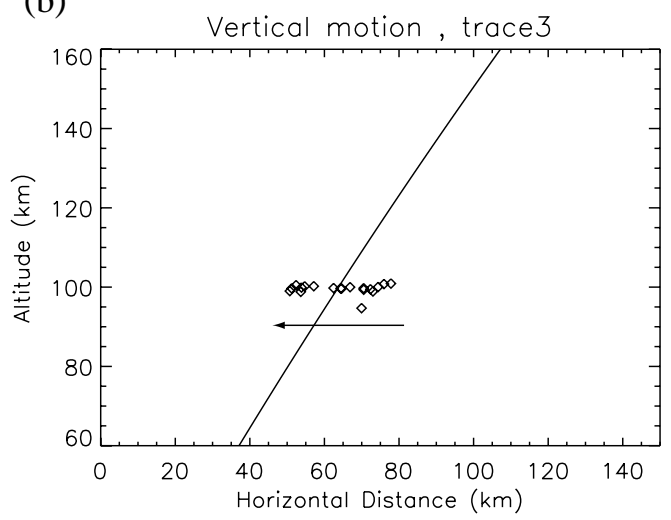

(d)

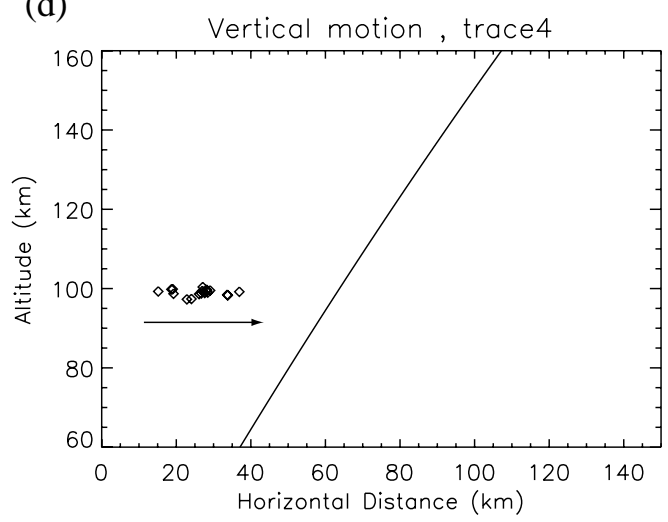

(c)

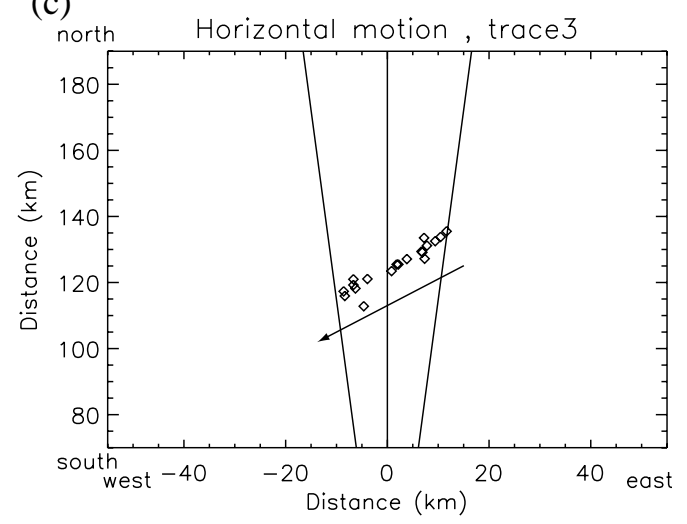

(e)

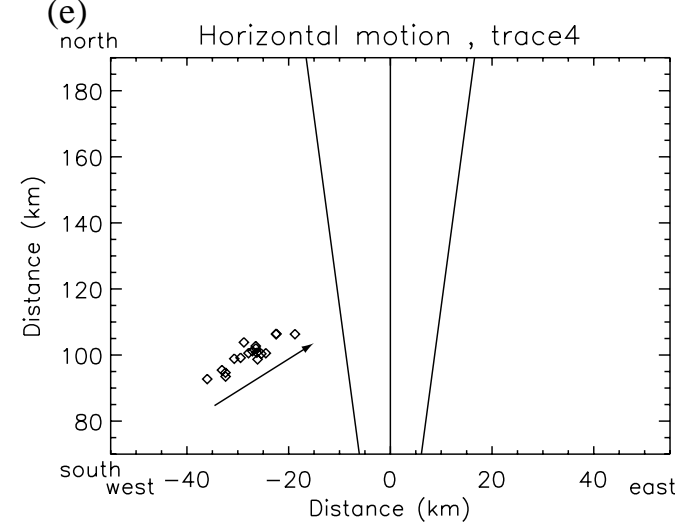

Fig. 9. Same as Fig. 8, except that 02 to 03 JST on 7 August 2002 are shown. (b) and (c) correspond to Trace 3, while (d) and (e) correspond to Trace 4.

(Larsen et. al., 2005), which was generally in agreement with our results derived from meteor echoes. The discrepancy between neutral winds derived from meteor echoes and the TMA trail may be due to the difference of observed regions.

Figure 11 plots the zonal and meridional components of neutral winds from the meteor echo observations (a) before and (b) after the reversal of range rate at 0240 JST on $7 \mathrm{Au}-$ gust 2002 as presented in Fig. 9(a). Horizontal bars on the neutral wind profiles are the standard deviations in the leastsquare estimation. The altitude resolution was reduced to $6 \mathrm{~km}$ in this plot because fewer meteor echoes were avail- able. There was a change in neutral wind at $103 \mathrm{~km}$ altitude from southeastward to northwestward corresponding to the reversal of range rate from negative to positive (Fig. 9(a)).

Table 3 compares neutral wind velocity at $100-103 \mathrm{~km}$ derived with the altitude resolution of $3 \mathrm{~km}$ and motion of the echoing region for $10 \mathrm{QP}-$-echo events observed from 3 to 8 August 2002. To infer the configuration of the echoing region, we prepared a model of the drifting echoing region and neutral wind. We assumed that the echoing regions were onedimensionally elongated or a chain of patches organized in a line drifting with local neutral wind. Neutral wind velocity 
Table 3. Relationship between direction of motion of echoing region and neutral wind and estimated directions of normal vectors of wavefronts. Angle is measured anti-clockwise from geographic east.

\begin{tabular}{lllcl}
\hline & Echoing Region (Range Rate) & Neutral Wind & Number of Events & Angle of FAI echo region $\left(\theta_{E}\right)$ \\
\hline (a) & Westward (Negative) & Southeastward & 5 & $1.8^{\circ}, 8.8^{\circ}, 5.2^{\circ}, 1.4^{\circ}, 2.3^{\circ}$ \\
(b) & Westward (Negative) & Northwestward & 2 & $75.3^{\circ}, 28.2^{\circ}$ \\
(c) & Eastward (Positive) & Southwestward & 1 & $7.2^{\circ}$ \\
(d) & Eastward (Positive) & Northeastward & 2 & $-13.5^{\circ}, 17.7^{\circ}$ \\
\hline
\end{tabular}
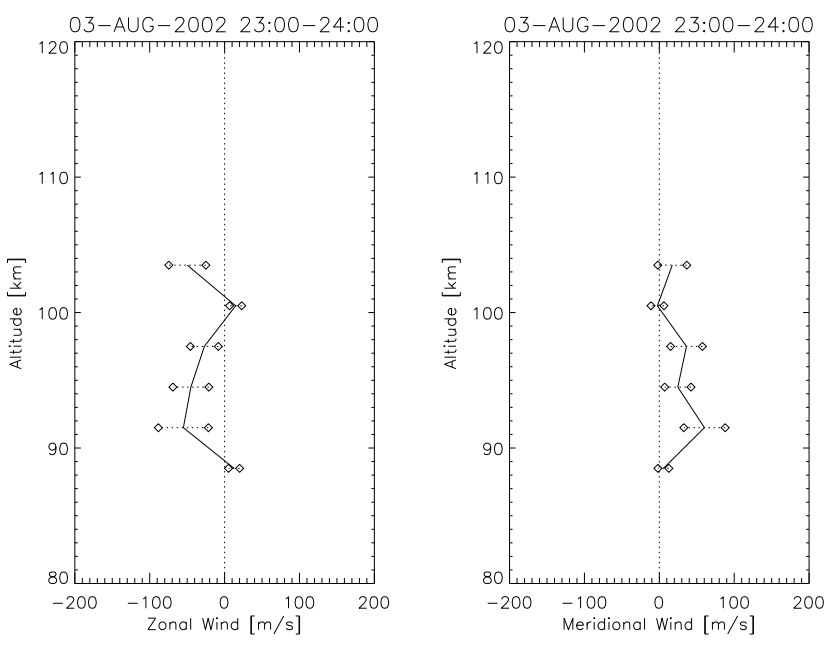

Fig. 10. Zonal (Eastward) and Meridional (Northward) components of neutral wind velocity from 2300, 3 August, to 0100, 4 August 2002 are shown.

was not necessarily perpendicular to the major axis of the echoing region. We also assumed that they move horizontally at a constant altitude. In Fig. 12, we showed the relationship between the true motion and the apparent motion to the radar for the echoing region elongated in various directions when southeastward wind was applied. We know direction of the apparent motion of the echoes $\left(\theta_{B}\right)$ from interferometry observations, and wind speed $\left(v_{n}\right)$ and its direction $\left(\theta_{W}\right)$ from simultaneous meteor observations. The true angle of the major axis of the echo region $\left(\theta_{E}\right)$ can be estimated as suggested in the figure. All angles are measured anti-clockwise from the geographic east. When the major axis of the echoing region is northwest-southeast (Fig. 12 (1) and (2)), the apparent motion of echoes is eastward if $\theta_{E}>\theta_{B}$, and westward if $\theta_{E}<\theta_{B}$. When the major axis of the echoing region is northeast-southwest, (Fig. 12 (3)-(5)) echoes will not move apparently if $\theta_{E}=\theta_{W}$, move eastward if $\theta_{E}>\theta_{W}$, and move westward if $\theta_{E}<\theta_{W}$. A similar model can be constructed for any neutral wind direction.

On 3 August 2002, when the rockets were launched, the estimated $\theta_{E}$ was $75^{\circ}$, that is, the estimated wavefront was from north-northwest to south-southeast. At this time, $v_{n}$ was $52 \mathrm{~m} / \mathrm{s}^{-1}$ to west-northwest which was very close to the (a)
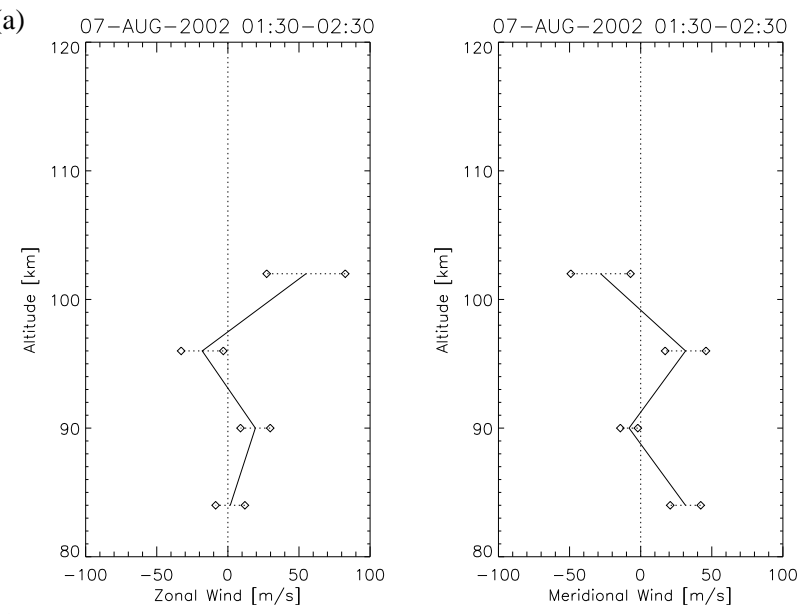

(b)
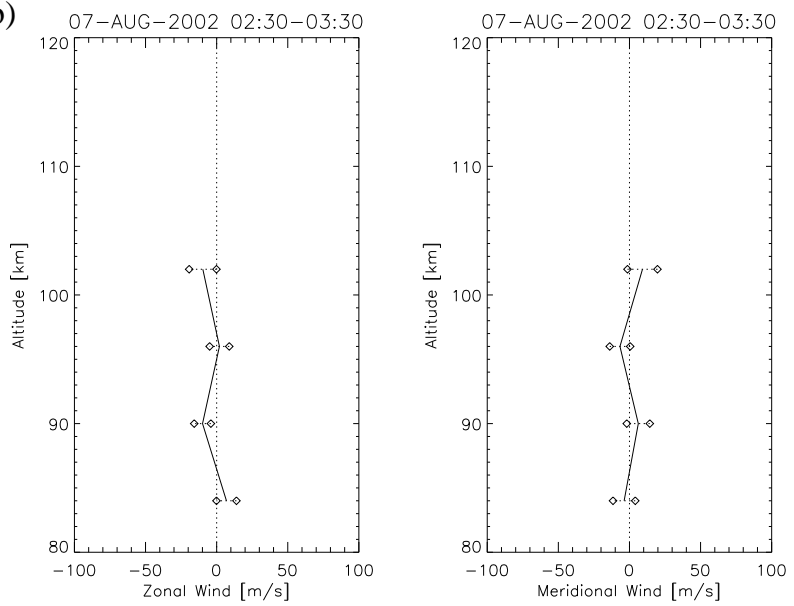

Fig. 11. Zonal (Eastward) and Meridional (Northward) components of neutral wind velocity (a) from 0130 to 0230 JST and (b) from 0230 to 0330 JST, 7 August 2002 are shown.

direction normal to the estimated wavefront. This orientation of the wavefront and its relationship to the neutral wind is the same as indicated by Fig. 12 (1). As the wind direction of this case is reversed from the figure, the motion of the echoing region is also reversed. Time delay of echo appearance of the LTPR behind the FAR is estimated from the observed wind velocity and $\theta_{E}$ as $18.3 \mathrm{~min}$. The delay is very close to what was directly measured from the cross correlation analysis of echo intensities (19.5 min, see Sect. 3). This reasonable 


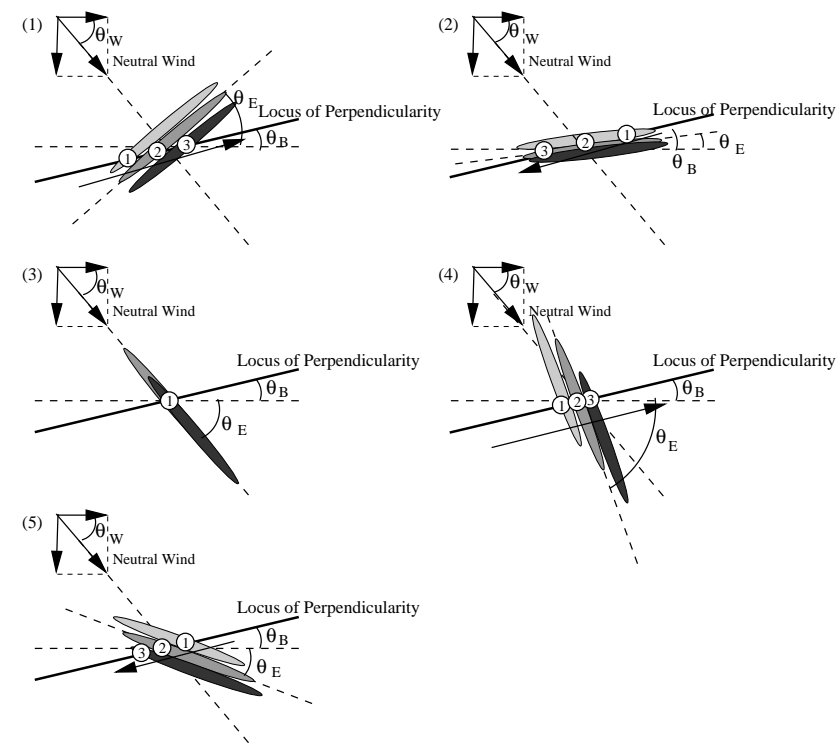

Fig. 12. How one-dimensionally elongated echoing region moves in the horizontal plane along locus of perpendicularity with neutral wind. Echoing region is assumed to be at constant altitude of $100 \mathrm{~km}$ moving with southeastward neutral wind. Angles are measured anti-clockwise from geographic east.

agreement validates our assumption for the analysis that linear wavefronts of FAIs are moving with neutral wind.

We wish to point out that winds measured by LTPR were used instead of those measured from the TMA trail (released during rocket upleg) although the latter measurements were geographically closer to the radar echoes than the former. If the TMA winds measured between 100 and $105 \mathrm{~km}$ were used, the results would not change significantly. This seems to justify using the neutral wind velocity derived from meteor echoes for this analysis. Having found that similar results could be obtained by using winds measured by LTPR in place of those from TMA technique, we proceeded to use LTPR winds for nights when TMA derived winds were not available.

We estimated $\theta_{E}$ for the ten events including 3 August 2002. The results are summarized in the fourth column of Table 3. In most cases, we estimated that $\theta_{E}$ were between $0^{\circ}$ and $20^{\circ}$. This means that the frontal structures are elongated east-west and are slightly tilted anti-clockwise. To summarize this, typically a range rate is negative (positive) when a frontal structure of echoing regions elongated eastnortheast-west-southwest drifts with southward (northward) neutral wind. This is consistent with the tendency for the echoes to appear at the FAR earlier than at the LTPR, which is located south of the FAR as mentioned in Sect. 3.

\section{Discussion}

Semidiurnal behavior of range rates for QP echoes was observed by Pan and Tsunoda (1999). They found that the range rates for QP echoes changed their signs from negative to positive around $22 \mathrm{LT}$ and from positive to negative again around $04 \mathrm{LT}$. They related this to the semidiurnal tide of lower thermospheric wind. Neutral wind measurements simultaneous with FAI observations have been done by TMA releases from sounding rockets to produce snapshots of neutral wind profiles. In the SEEK-2 campaign, we observed FAIs and neutral wind continuously, which made it possible for the first time to compare QP echoes and neutral wind behavior. Between 02 and 03 JST on 7 August 2002, reversal of the range rate was observed as we can see from Fig. 9(a). The reversal occurred at $0230 \mathrm{JST}$, which is even later than the 22 LT reported by Pan and Tsunoda (1999), coinciding with the change of neutral wind. Throughout the SEEK-2 observation, such reversal after 22 LT was not clear, and negative range rates were very often observed later than 22 JST. From our observations, we knew that a negative (positive) range rate corresponded to westward (eastward) motion along the locus of perpendicularity. This is apparent motion depending on the shape and direction of motion for the echoing region as discussed in the previous section.

Recently, Hysell et al. (2004) conducted simultaneous observation of $E_{s}$ layers and QP echoes by the Arecibo incoherent scatter radar and a $30-\mathrm{MHz}$ coherent scatter radar. They found patchy structures of $E_{s}$ forming wavefronts by the incoherent scatter radar when the QP echoes were observed by the coherent scatter radar. They also found bands of echoing region elongated in the east-west direction by the coherent scatter radar. The band structure found by them could corresponds to the wavefront inferred by our analysis. Larsen (2000) argued that the horizontal wavelengths of Kelvin-Helmholtz structure could be $\sim 8-16 \mathrm{~km}$. The size of the patches of echoing regions observed by the LTPR was $10-30 \mathrm{~km}$ as shown in Figs. 8 and 9. By observing FAIs and neutral wind simultaneously with interferometry measurements, we showed that the motion of these structures were closely related to the background neutral wind by simultaneous FAI and meteor echo observations.

Yamamoto et al. (1997) showed that wavefronts of QP echoes were aligned with the direction from northwest to southwest and propagated southwestward, which were different from our results that the wavefronts were often aligned east-northeast-west-southwest. They used the MU radar and a portable VHF radar separated by approximately $40 \mathrm{~km}$ and compared the RTI plots by non-interferometry observation. Since analysis was done for the data in relatively short period, ten events in six nights from 3 to 8 August 2002, possibility for the wavefronts to be aligned in northwestsoutheast should not be excluded.

The instability of $E_{s}$ layer proposed by Cosgrove and Tsunoda (2002) suggests that the growth rate maximizes when the wave front is in northwest-southeast direction. The growth rate shown by them are nearly zero or slightly negative for the typical wave fronts obtained by our analysis which are aligned in east-northeast to west-southwest. Onoma et al. (2005) observed atmospheric gravity waves (AGWs) at altitudes of approximately $86 \mathrm{~km}$ and $96 \mathrm{~km}$ altitudes using an airglow imager located near the FAR site 
from 1 to 8 August 2002. They found that a dominant direction atmospheric gravity waves propagated was southeastward when FAIs were observed by the LTPR. Our findings where the frontal structures of FAIs were predominantly eastnortheast to west-southwest direction seem to indicate the importance of the AGWs in the generation process of $\mathrm{QP}$ echoes.

\section{Conclusion}

We conducted observations of FAI echoes, especially QP echoes, associated with midlatitude sporadic-E layers in the southwest of Japan with the LTPR and the FAR. The radars were located $40 \mathrm{~km}$ apart and utilized together with two rocket experiments. The LTPR measured FAIs and neutral wind simultaneously and continuously by interferometry measurements. the LTPR and the FAR very often provided similar shapes for QP echoes with certain lag times. Echoes typically appeared 10 min earlier at the FAR than at the LTPR. This suggests that plasma irregularities associated with $E_{s}$ layers are not distributed randomly but are organized with a correlation length of more than several tens of kilometers.

One of the important findings is that QP structures of the FAI echoes were horizontal in this experiment. We also find that the QP structures are well explained by the spatial variability and motion of the neutral atmosphere. By tracking echoes of QP striations, we confirmed the suggestion by Hysell et al. (2002) that echoes would come from side lobes in addition to the main lobe. It was clear that QP striations on RTI plots were produced by the horizontal motion of echoing regions across the field of view of the radar along the locus of perpendicularity. Few echoes were detected at altitudes higher than $120 \mathrm{~km}$ or lower than $90 \mathrm{~km}$. QP echoes with negative range rates appeared on the eastern side lobes at longer ranges. As they moved westward along the locus of perpendicularity at constant altitude with decreasing range through the main lobe to the western side lobes, which was still to the east of geomagnetic north, distances to the scattering points decreased. Echoes with positive range rates, on the other hand, moved eastward. Echoing regions seem to be one-dimensionally elongated, and often patchy and aligned.

QP echoes have been reported to propagate often southeastward by previous studies (e.g. Yamamoto et al., 1997). The $E_{s}$ layer instability mechanism proposed by Cosgrove and Tsunoda (2002) is most effective in this direction. By analyzing movement of the echoes and the neutral wind during the SEEK-2 period, however, we found that the echoing region was aligned north-northeast to south-southwest propagating west-northwestward on 3 August 2002, when the rockets were launched. And it was often, eight out of ten QP events in the present study, aligned east-northeast to west-southwest. This shows that QP echoes can be observed not only when the echoing regions are aligned northwest to southeast as suggested by Cosgrove and Tsunoda (2002). This was made possible for the first time by the simultaneous observations of neutral wind and FAIs with interferometry measurements. Onoma et al. (2005) reported from their airglow observations that a predominant direction of propagation of atmospheric gravity waves was southeastward when QP echoes were observed. Their result is consistent with ours. This finding indicates that AGW seeding of QP echoes cannot be ignored. In practice, AGWs, the $E_{S}$ layer instability, and the shear instability (Larsen, 2000) may intricately interrelated with each other. To distinguish between those effects, more extensive simultaneous observations of FAIs, neutral wind, and AGWs are necessary.

Acknowledgement. This study was supported in part by a Grantin-aid for Scientific Research B(2)(14340145) made by the Ministry of Education, Science, Sports and Culture, Japan. The authors are grateful to Nishinoomote city, Kagoshima prefecture, Japan, for their kind support in operating the FAR, and to the Japanese Aerospace Exploration Agency for their kind support in operating the LTPR.

Topical Editor M. Pinnock thanks J. L. Chau and another referee for their help in evaluating this paper.

\section{References}

Bernhardt, P. A.: The modulation of sporadic-E layers by KelvinHelmholz billows in the neutral atmosphere, J. Atmos. SolarTerr. Phys., 64, 1487-1504, 2002.

Cosgrove, R. B. and Tsunoda, R. T.: A direction dependent instability of sporadic-E layers in the nighttime midlatitude ionosphere, Geophys. Res. Lett., 29, 11-1, doi:1029/2002GL014669, 2002.

Fukao, S., Yamamoto, M., Tsunoda, R. T., Hayakawa, H., and Mukai, T.: The SEEK (Sporadic-E Experiment over Kyushu) campaign, Geophys. Res. Lett., 25, 1761-1764, 1998.

Hysell, D. L. and Burcham, J. D.: The 30-MHz radar interferometer studies of midlatitude E region irregularities, J. Geophys. Res., 105, 12 797-12 812, 2000.

Hysell, D. L., Yamamoto, M., and Fukao, S.: Imaging radar observations and theory of type I and type II quasi-periodic echoes, J. Geophys. Res., 107A11, 1360, doi:10.1029/2002JA009292, 2002.

Hysell, D. L., Larsen, M. F., and Zhou, Q. H.: Common volume coherent and incoherent scatter observations of mid-latitude sporadic E-layers and QP echoes, Ann. Geophys., 22, 3277-3290, 2004 , SRef-ID: 1432-0576/ag/2004-22-3277.

Larsen, M. F.: A shear instability seeding mechanism for quasiperiodic radar echoes, J. Geophys. Res., 105, 24 931-24 940, 2000.

Larsen, M. F., Yamamoto, M., Fukao, S., Saito, A., and Tsunoda, R. T.: SEEK 2: Observations of neutral winds, wind shears, and wave structure during a sporadic E/QP event, Ann. Geophys., 23 , 2369-2375, 2005

Maruyama, T., Fukao, S., and Yamamoto, M.: A possible mechanism for echo striation generation of radar backscatter from midlatitude sporadic E, Radio Sci., 35, 1155-1164, 2000.

Miller, K. L. and Smith, L. G.: Incoherent scatter radar observations of irregular structure in mid-latitude sporadic E layers, J. Geophys. Res., 83, 3761-3775, 1978.

Ogawa T., Takahashi, O., Otsuka, Y., Nozaki, K., Yamamoto, M., and Kita, K.: Simultaneous middle and upper atmosphere radar and ionospheric sounder observations of midlatitude $\mathrm{E}$ region 
irregularities and sporadic E layer, J. Geophys. Res., 107A10, 1275, doi:10.1029/2001JA900176,, 2002.

Onoma, F., Otsuka, Y., Shiokawa, K., Ogawa, T., Yamamoto, M., Fukao, S., and Saito, S.: Relationship between gravity waves in $\mathrm{OH}$ and $\mathrm{OI}$ airglow images and VHF radar backscatter from E-region filed-aligned irregularities during SEEK-2 campaign, Ann. Geophys., 23, 2385-2390, 2005.

Pan, C. J. and Tsunoda, R. T.: Semidiurnal behavior of quasiperiodic echoes in the mid-latitude $E_{S}$ region observed with the Chung-Li VHF radar, Geophys. Res. Lett., 26, 2621-2624, 1999.

Tsunoda, R. T., Fukao, S., and Yamamoto, M.: On the origin of quasi-periodic radar backscatter from midlatitude sporadic E, Radio Sci., 29, 349-365, 1994.

Tsunoda, R. T., Cosgrove, R. B., and Ogawa, T.: Azimuthdependent $E_{S}$ layer instability: A missing link found, J. Geophys. Res., 109A12303, doi:1029/2004JA010597, 2004.

Wang, C.-H. and Chu, Y.-H.: Interferometry investigations of bloblike sporadic E plasma irregularity using the Chung-Li VHF radar, J. Atmos. Solar-Terr. Phys., 63, 123-133, 2001.

Woodman, R. F., Yamamoto, M., and Fukao, S.: Gravity wave modulation of gradient instabilities in mid-latitude sporadic E irregularities, Geophys. Res. Lett., 18, 1197-1200, 1991.
Yamamoto, M., Fukao, S., Woodman, R. F., Ogawa, T., Tsuda, T., and Kato, S.: Mid-latitude E region field-aligned irregularities observed with the MU radar, J. Geophys. Res., 96, 15943$15949,1991$.

Yamamoto, M., Kodama, N., Fukao, S., Tsunoda, R. T., Ogawa, T., and Tsuda, T.: Spatial structure of the E region field-aligned irregularities revealed by the MU radar, Radio Sci., 29, 337-347, 1994.

Yamamoto, M., Fukao, S., Tsunoda, R. T., Igarashi, K., and Ogawa, T.: Preliminary results from joint measurements of E-region field-aligned irregularities using the MU radar and the frequencyagile radar, J. Atmos. Terr. Phys., 59, 1655-1663, 1997.

Yamamoto, M., Fukao, S., Tsunoda, R. T., and Hayakawa, H.: SEEK-2 (Sporadic-E Experiment over Kyushu II) - Project Outline and Significance -, Ann. Geophys., 23, 2295-2305, 2005

Yokoyama, T., Yamamoto, M., and Fukao, S.: Computer simulation of polarization electric fields as a source of midlatitude field-aligned irregularities, J. Geophys. Res., 108(A2), 1054, doi:10.1029/2002JA009513, 2003.

Yokoyama, T., Yamamoto, M., Fukao, S., and Cosgrove, R. B.: 3$\mathrm{D}$ simulation on generation of polarization electric field in the midlatitude E-region ionosphere, J. Geophys. Res., 109A01309, doi:10.1029/2003JA010238, 2004. 